\title{
DELAY DIMINISHED EFFICIENT TASK SCHEDULING AND ALLOCATION FOR HETEROGENEOUS CLOUD ENVIRONMENT
}

\author{
Dr. N. Bhalaji, \\ Associate Professor, Department of Information Technology, \\ SSN College of Engineering, Chennai. \\ Email id: bhalajin@ssn.edu.in
}

\begin{abstract}
Cloud computing being a promising paradigm has become very prominent among a wide range of applications due to their timely service rendering capability. Attracted to the type of servicing and the way of servicing lots and lots of users, adapt to the cloud computing. This makes the time servicing of the cloud computing a tedious job. So in order to effectively handle the tasks the scheduling approach is entailed in the cloud computing. The paper proposes an efficient task scheduling for the heterogeneous cloud to render service at a minimized delay utilizing the genetic algorithm. The proposed method is validated through the, cloud simulator to understand the efficiency of the same in terms of delay and the quality of service.
\end{abstract}

Keywords: cloud computing, task scheduling, minimized delay, quality of service, heterogeneous networks.

\section{INTRODUCTION}

The scheduling problem is a NP -complete problem [1] as it requires complex steps in solving them meeting all the requirements as the make span, cost, energy efficiency etc. The task scheduling process which is an incorporated component into the computing does the reducing of the overall task into subtask and effectively assign them to the resources available.

The cloud computing that seems to very promising in availing various services to its users based on the requisition. The demand based servicing and the timely access enabled by the cloud makes it more prominent among a wide range of applications. It ubiquitous nature and ability to adopt to heterogeneous environment makes the cloud computing even more popular and unavoidable which makes enormous set of applications to switch to the cloud computing.

Apart from its nature of servicing the services provided by the cloud makes them even more attractive among enormous of applications. As they enable an elastic service that is scalable and evade the necessity of the capital investment and the maintenance cost by providing the software, infrastructure and the platform as service, due all these merits of cloud computing more and more users get added, day by day to the cloud computing making it's processing very slow. So this could be handled by scheduling of the tasks that arrive to the cloud. 
Journal of trends in Computer Science and Smart technology (TCSST) (2019)

Vol.01/No.01

Pages: 46-56

https://irojournals.com/tcsst/

DOI: https://doi.org/10.36548/jtcsst.2019.1.005

So there arises a need for an efficient task scheduling that function's managing the delay incurred in the service rendering of the cloud computing. As there are many research works proceeded in having an effective tasks scheduling there are still few demerits in the approaches of scheduling.

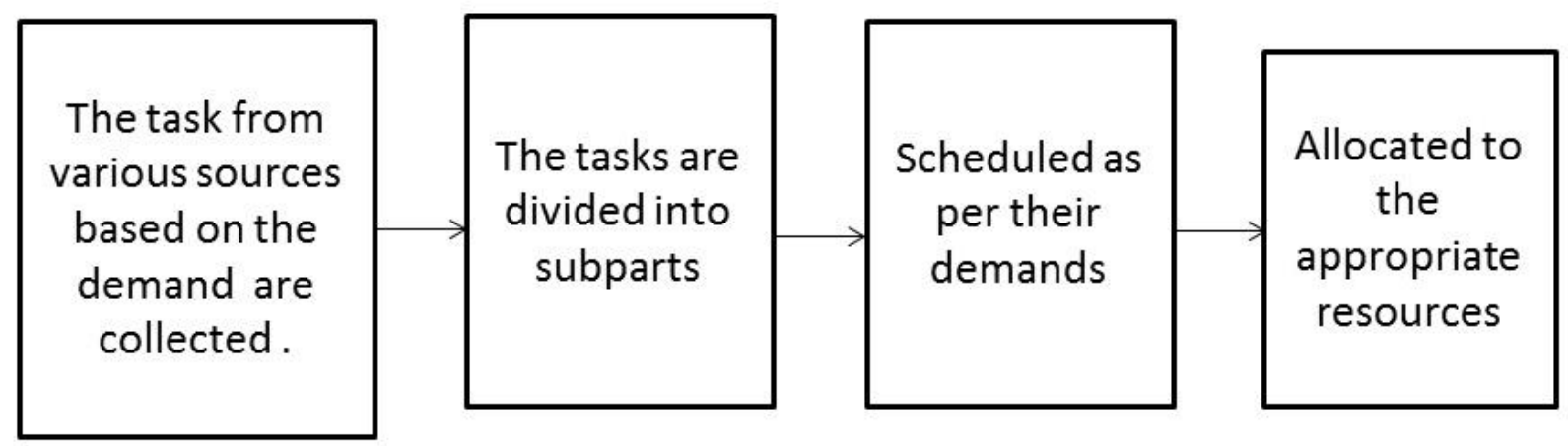

Fig .1 The Task Scheduling Process

So the proposed method does an effective task scheduling, to reduce the delay incurred in the service provisioning, this method also seems to energy efficient as it takes the energy utilized also into consideration.

The remaining of the paper is organized with the 2. Related works. 3 Proposed work of efficient task scheduling that has less delay. 4. The result evaluation analyzing the efficiency of the scheduling, 5. Conclusion.

\section{RELATED WORKS}

Topcuoglu et al [1] presents the heterogeneous EFT algorithm for scheduling as to have an optimized speed, make span etc. He et al [2] proposes a novel scheduling for the tasks for the grid and the ubiquitous computing utilizing the general adaptive scheduling heuristics. Lakra et al [3], address the throughput optimization in the service rendering of the cloud by proper scheduling of the tasks in the data centers to improve the throughput and the reduce the cost. Patel et al [4], utilizes the min-min algorithm for the task scheduling identifying the tasks with the minimum time and allocating it to the appropriate resources, thereby reducing the cost of utilization and improving the throughput of the system. Pham et al [5] introduces the task scheduling in the cloud-fog computing to improve the tradeoff by employing a heuristic based algorithm. This causes an improved in the makes span for the users of the large scale applications. Bittencourtet al [6] proposes the task scheduling in the fog computing, which takes an efficient alternative role of the cloud computing Man et al[7] the paper proposes the scheduling model that is based 
Journal of trends in Computer Science and Smart technology (TCSST) (2019)

Vol.01/No.01

Pages: 46-56

https://irojournals.com/tcsst/

DOI: https://doi.org/10.36548/jtcsst.2019.1.005

on the cost and the finish time providing a balance in the cost and the scheduling. Krishnadoss et al [8] the paper presents a task scheduling algorithm by using the OSCA algorithm to have a minimized cost and the make span. Park, et al [9] the author proposes the optimal scheduling algorithm that is based on task duplication to have an efficient task scheduling that is experiences an improved through put among the distributed memory machines. Salim et al [10] presents a task scheduling algorithm for the processor of the heterogeneous types. Koehler et al [11] the author details the service provisioning of the cloud and the reason for the attractiveness towards the cloud. The service provisioning way makes the cloud very popular among a wide range of applications. Casas, et al [12] the paper presents the popularity gained by the cloud computing and the reason behind it, and the different services provided by the cloud and so on This benefits promised by the cloud computing make them very prominent among a wide range of applications and increase's the necessity of the task scheduling though the multitude of task scheduling prevail there is none that performs the scheduling satisfying all the requirement of the users. So in the proposed method we utilize the adaptive heuristic evolutionary algorithm-genetic algorithm that is a search based on the natural selection to address the problem of the task scheduling in the heterogeneous cloud environment Whitley, et al [13], and Davis, [14] presents the basics of the genetic algorithm. The proposed methodology utilizing the genetic algorithm is evaluated along with the PSO, and other task scheduling algorithms to know the efficient delay management of the present system.

\section{PROPOSED WORK}

The cloud computing which is a promising paradigm for a wide range of applications due to its inbuilt benefits such as the efficiency in the way of providing the services, such as the software as service, platform as service, and the infrastructure as the service. This enables the users to elude the unnecessary expenditure such as the capital investment and the licensing. The services are provides with a better scalability minimizing the in-house operational issues, elasticity, ability to share the resources with the multiple users, anywhere, anytime access, the prevention measures in the losses incurred due to the natural disasters and the outages in the power. This causes an immeasurable impact on the business leading many companies to shift to the cloud computing. The figure 2 below gives the few impacts of the cloud computing in a business. 


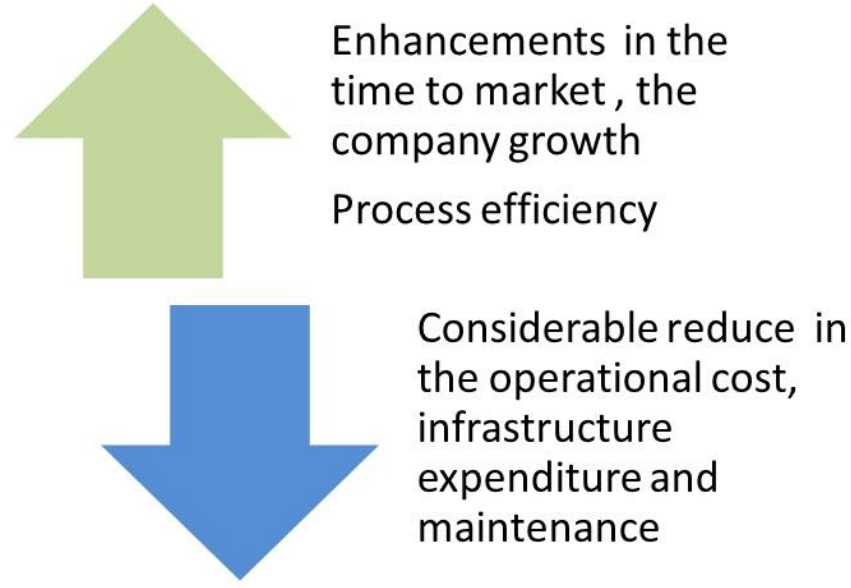

Fig .2 Impacts of a cloud computing in a Business

All the impacts shown in the fig . 2 above make them very prominent among a wide range of applications and also overloaded paving way for the task scheduling. The task scheduling based on the genetic algorithm is proposed in the paper to have effective way of resource allocation to the higher priority tasks that have a limited time for its completion. The tasks received as seen in fig .1 is split into multiple tasks and each task has to be allocated to the appropriate resources as the each virtual machines takes different time for the processing of the tasks. So there allocation of the task to the appropriate virtual machine is essential to have a task schedule that has a diminished delay.

\subsection{PROBLEM DEFINITION}

The problem of task scheduling is framed as an undirected graph $G(V, E)$ where the tasks to be allotted are the represented as the $V$ and the precedence constraints of the tasks are referred as the $E$ edges. The virtual machines to be allocated for each task are given as the $V_{M}=\left\{V_{m 1}, V_{m 2}, \ldots \ldots V_{m i}\right\}$. The proposed method applies the genetic algorithm in the allocation of the resources to the tasks, enumerating the more efficient virtual machines that are considered to be optimal for the completion of the tasks within the time. Thus reducing the delay incurred in the scheduling of the tasks. 


\subsection{THE STRIDES IN THE SCHEDULING OF THE TASK}

Before proceeding with the scheduling of the tasks, the tasks are split into sub parts, and the information regarding the start time $\left(S_{\text {time }}\right)$, end time $\left(E_{\text {time }}\right)$ and the processing time $\left(P_{\text {time }}\right)$ along with the information of its parent or child if any them is available is gathered and subjected to the scheduling of the task. These information's are utilized in estimating the completion time of the whole task. The equation.1 below provides the time required for the calculation of the completion time of the whole task.

$$
\text { Comp }_{\text {time }}=\Sigma\left(P_{\text {timei }}\left(\frac{I_{s i}+O_{s i}}{B_{w i}}\right)\right)
$$

Where the $I_{s i}$ and the $O_{s i}$ is possible input and the output sizes of each individual sub tasks and the $B_{w i}$ provide the band width of the network.

Once the completion time is calculated the tasks are scheduled by prioritizing based on their completion time and allocated to the appropriate virtual machines, before allocation of the resources the resources are evaluated for selecting an optimal one based on the process of natural selection applying the genetic algorithm.

\subsection{OPTIMAL VM SELECTION USING THE GENETIC ALGORITHM}

As the allocation of the resources play a major role in the process of scheduling for the timely completion of the task according to the user requirements. It becomes necessary for the evaluation of the optimal resources for the easy and the early completion of the tasks. The genetic algorithm with the natural process of the selection, mutation and crossover decides with the optimal resources based on the time taken by it to complete a task, the cost of the resource and the average power consumed by the resource. For a set of virtual machines available in the network it time taken to complete the task $\left(C_{t}\right)$, its power consumption $\left(P_{c}\right)$ and the cost $\left(A_{c}\right)$ are gathered, and the optimal resources are achieved after performing the evaluation based on the genetic algorithm. The process continues for multiple iterations to converge at an optimal resources that are more suitable for the tasks. The fig. 3 below shows the proposed task scheduling architecture. 
Journal of trends in Computer Science and Smart technology (TCSST) (2019)

Vol.01/No.01

Pages: 46-56

https://irojournals.com/tcsst/

DOI: https://doi.org/10.36548/jtcsst.2019.1.005

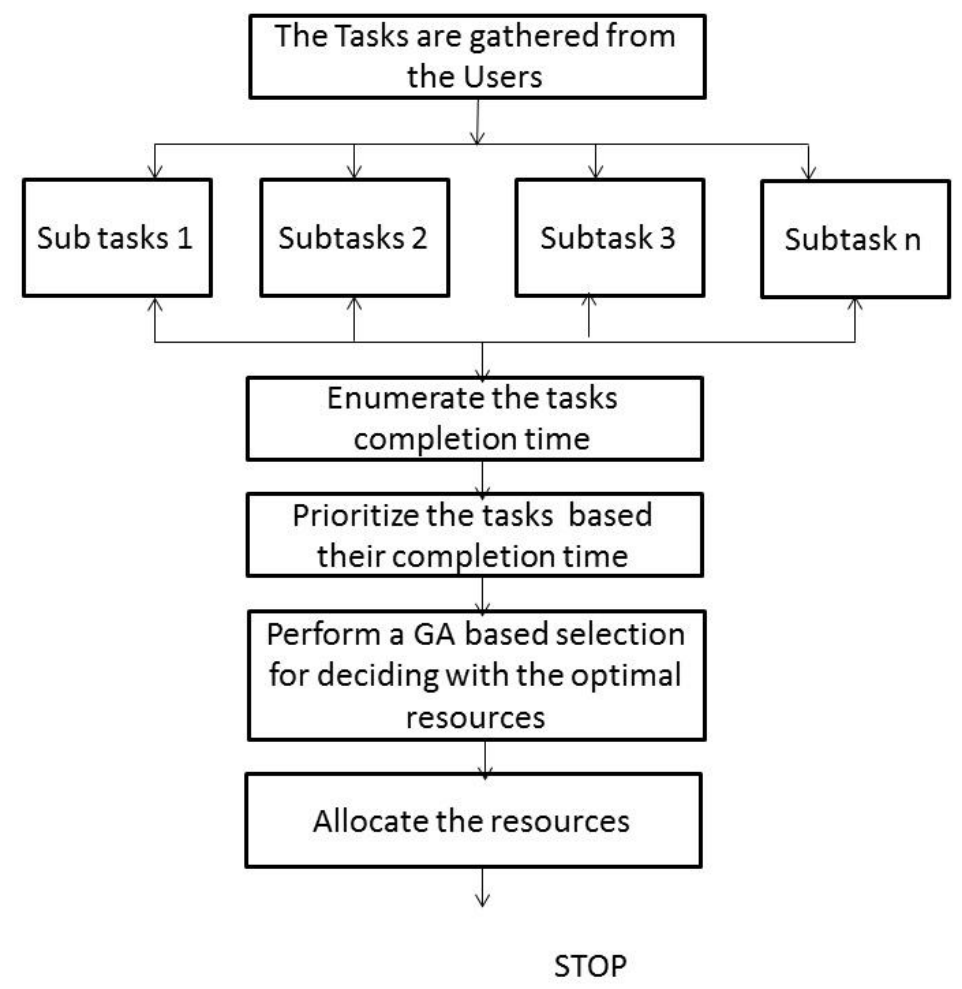

Fig.3 The Proposed Process of Task Scheduling

\subsection{THE PROPOSED ALGORITHM OF TASK SCHEDULING AND ALLOCATION}

The steps in efficient task scheduling and the allocation using the genetic algorithm are as follows.

Step1: The set of tasksT $=\left\{t_{1}, t_{2} \ldots \ldots . t_{n}\right\}$ are collected from the users, divided into subtask.

Step2: The for each sub tasks $S T=\left\{S T_{1}, S T_{2} \ldots \ldots \ldots S T_{3}\right\}$ the completion time is determined using the equation (3) Step3: The Estimated Tasks are prioritized based on the completion time, in the ascending order with the least completion time occupying the initial position.

Step4: For each $V_{M}=\left\{V_{m 1}, V_{m 2}, \ldots \ldots V_{m i}\right\}$ the $C_{t}, P_{c}, A_{c}$ are gathered.

Step5: The Genetic Algorithm is applied to decide with the perfect resources that are suitable for the tasks.

Step6: The fitness of the each $V_{M}$ is evaluated based on the $C_{t}, P_{c}, A_{c}$ to identify the tasks with the $M I N\left(C_{t}, P_{c}, A_{c}\right)$

Step7: Performs the mutation with the rate of .2 and the cross over with the rate of 5

Step8: For the new set of population generated evaluates fitness and converges with the optimal resources.

Step 9: After the achieving the optimal set of resources, it stops 
Journal of trends in Computer Science and Smart technology (TCSST) (2019)

Vol.01/No.01

Pages: 46-56

https://irojournals.com/tcsst/

DOI: https://doi.org/10.36548/jtcsst.2019.1.005

\section{RESULTS}

The results obtained by evaluating the present system in the cloudsim, for different set of tasks ranging from 100 to 500 , with the initial energy of 1000 joules, with the simulation time of 100 seconds, with the packets size of 2400 bytes. The proposed process of task scheduling and allocation with the genetic algorithm and the other methods of PSO and the SA are evaluated in the cloudsim to understand the efficiency of the proposed method in terms of the delay, energy consumption and the cost reduction.

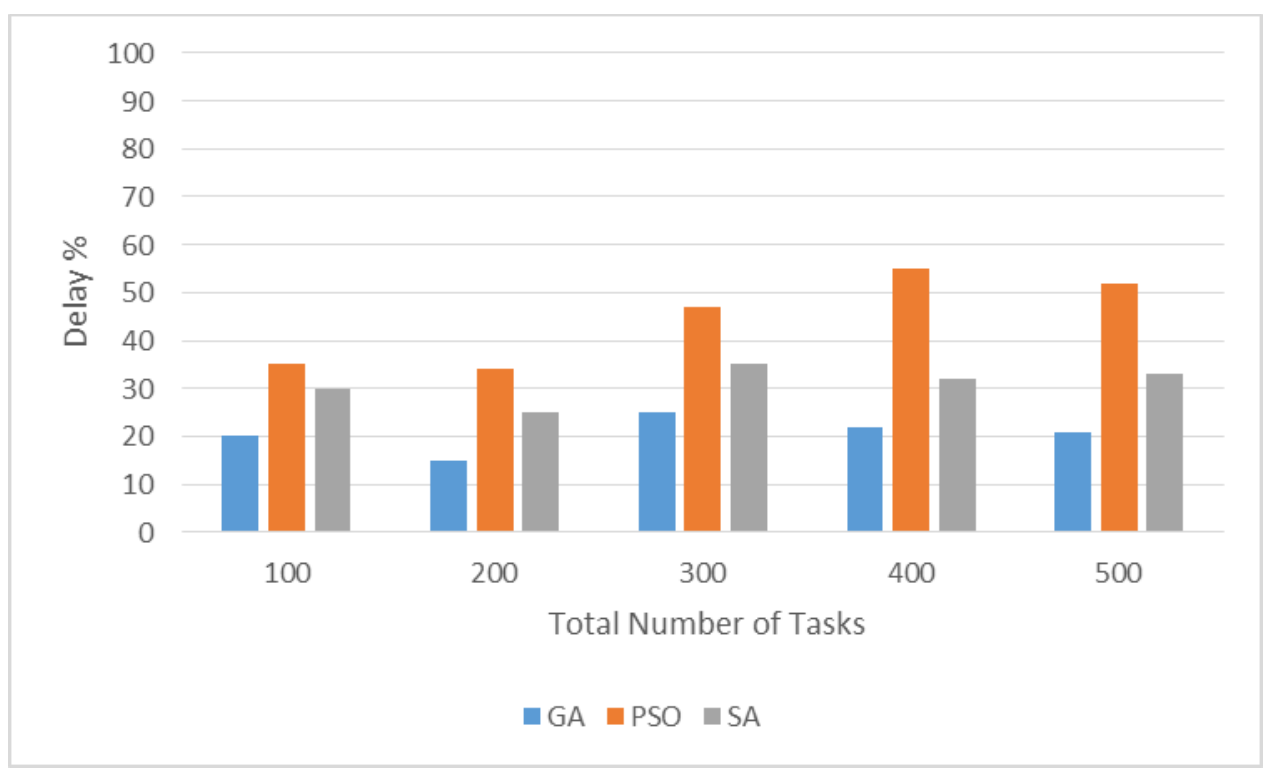

Fig.4 Percentage of Delay Incurred

The fig. 4 shows the percentage of the delay obtained in the completion of the complete task, that includes the scheduling of the task, allocation and the time taken in the completion of the of the task. The equation below shows the different process where the delay could incurred when handling a task.

$$
\text { Delay }_{\text {incurred }}=S c h_{\text {task }}+\text { Allo }_{\text {Resour }}+\text { time }_{\text {resour }}
$$

Where the $S c h_{\text {task }}$ is the time taken in the scheduling of the task, Allo Resour $_{\text {is }}$ is the time take in the allotion of the resource and the time resour $_{\text {is }}$ the time taken by the resources to complete the task. The proposed method utilizing 
Journal of trends in Computer Science and Smart technology (TCSST) (2019)

Vol.01/No.01

Pages: 46-56

https://irojournals.com/tcsst/

DOI: https://doi.org/10.36548/jtcsst.2019.1.005

the genetic algorithm in scheduling as well as the allocation enables to have an optimal scheduling and allocation that reduces the time consumed in the process of the scheduling and allocation by the other methods.

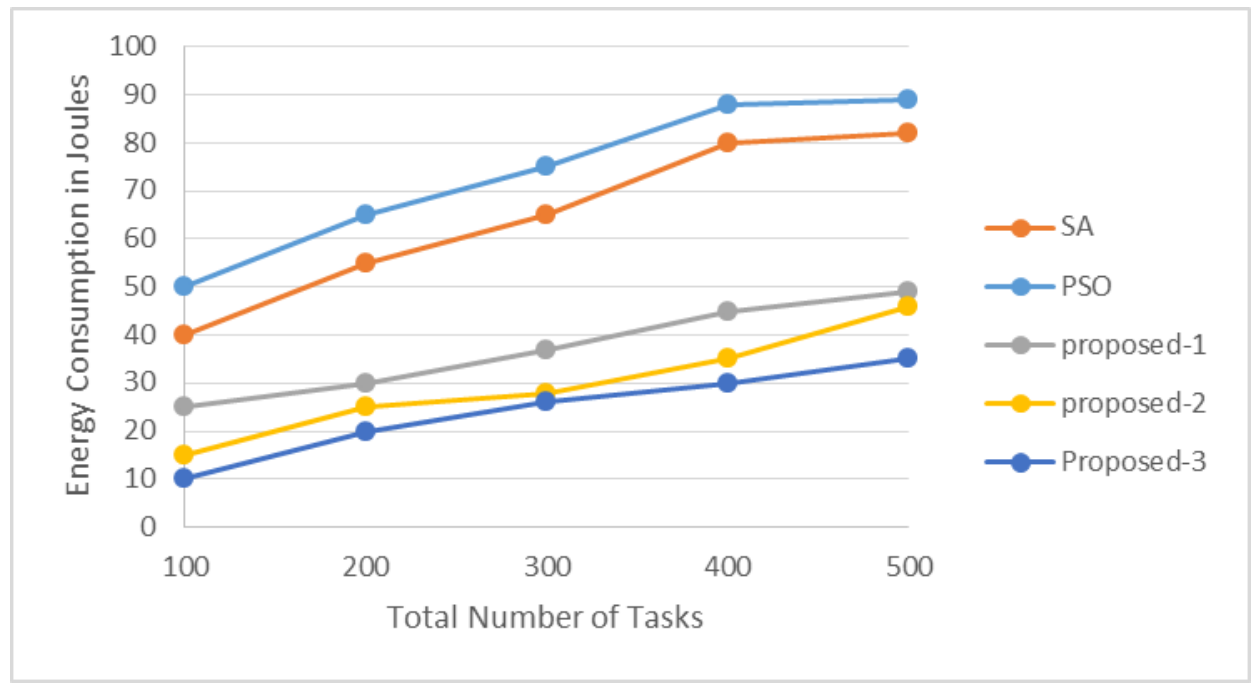

Fig .5 Energy Consumption

The fig.5 shows the result obtained for the proposed method on the energy consumption of the process of the scheduling and the allocation, since the proposed method enumerates the resources with the limited energy consumption, the average energy consumption of the task scheduling and allocation utilizing the genetic algorithm is very much reduced compared to the other methods such as the PSO and the SA. 
Journal of trends in Computer Science and Smart technology (TCSST) (2019)

Vol.01/No.01

Pages: $46-56$

https://irojournals.com/tcsst/

DOI: https://doi.org/10.36548/jtcsst.2019.1.005

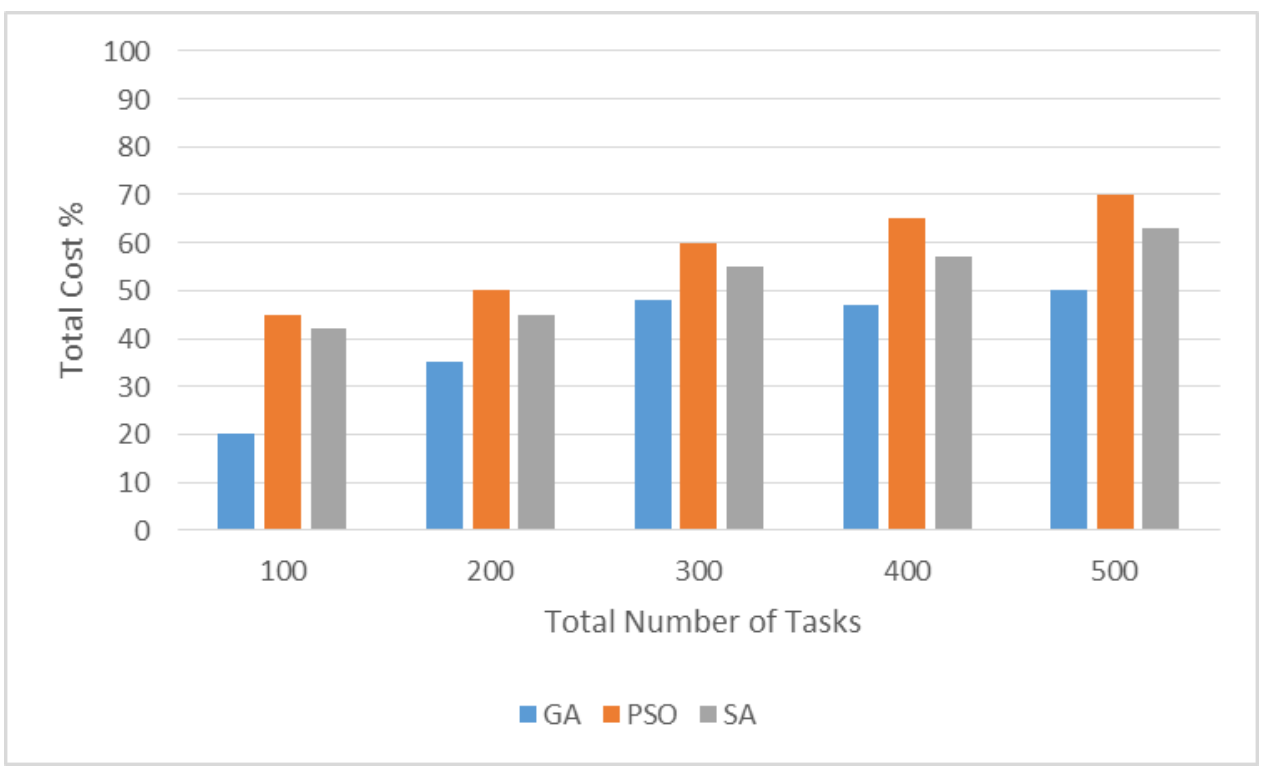

Fig .6 Total Cost

The fig 6 shows the percentage of cost reduced by the proposed method of task scheduling and allocation compares to the other methods, particle swarm optimization (PSO) and the simulated annealing (SA). Since the scheduling based on the processing time of the resources once the resource completes a task it is immediately allocated with the next prioritized tasks. As the VM in cloud are charged in hourly bases, the allocation of the resources with the high processing speed through seems to be costly, helps in completion of more number of tasks, compared to the allocation of the tasks to the resources with the low operating speed.

\section{CONCLUSION}

The paper proposes an efficient task scheduling and the allocation that is processing at a diminished delay and improved quality of service. For this the paper utilizes the genetic algorithm that arrives at an optimal solution utilizing the natural process of selection. The genetic algorithm in the proposed method is employed both in the selection of the optimal resources for scheduling and the allocation of the resources to the tasks, enumerating the resources that are efficient based on the processing speed, average power consumption and the cost. Further the same is evaluated in the cloudsim to evince the efficiency of the proposed delay diminished task scheduling and the allocation. 
Journal of trends in Computer Science and Smart technology (TCSST) (2019)

Vol.01/No.01

Pages: 46-56

https://irojournals.com/tcsst/

DOI: https://doi.org/10.36548/jtcsst.2019.1.005

\section{REFERENCES}

[1] Topcuoglu, Haluk, Salim Hariri, and Min-you Wu. "Performance-effective and low-complexity task scheduling for heterogeneous computing." IEEE transactions on parallel and distributed systems 13, no. 3 (2002): 260-274.

[2] He, XiaoShan, XianHe Sun, and Gregor Von Laszewski. "QoS guided min-min heuristic for grid task scheduling." Journal of Computer Science and Technology 18, no. 4 (2003): 442-451.

[3] Lakra, Atul Vikas, and Dharmendra Kumar Yadav. "Multi-objective tasks scheduling algorithm for cloud computing throughput optimization." Procedia Computer Science 48 (2015): 107-113.

[4] Patel, Gaurang, Rutvik Mehta, and Upendra Bhoi. "Enhanced load balanced min-min algorithm for static meta task scheduling in cloud computing." Procedia Computer Science 57 (2015): 545-553.

[5] Pham, Xuan-Qui, and Eui-Nam Huh. "Towards task scheduling in a cloud-fog computing system." In 2016 18th Asia-Pacific network operations and management symposium (APNOMS), pp. 1-4. IEEE, 2016.

[6] Bittencourt, Luiz F., Javier Diaz-Montes, Rajkumar Buyya, Omer F. Rana, and Manish Parashar. "Mobility-aware application scheduling in fog computing." IEEE Cloud Computing 4, no. 2 (2017): 26-35.

[7] Man, Nguyen Doan, and Eui-Nam Huh. "Cost and efficiency-based scheduling on a general framework combining between cloud computing and local thick clients." In 2013 International Conference on Computing, Management and Telecommunications (ComManTel), pp. 258-263. IEEE, 2013.

[8] Krishnadoss, Pradeep, and Prem Jacob. "OCSA: task scheduling algorithm in cloud computing environment." Int J Intell Eng Syst 11, no. 3 (2018): 271-279.

[9] Park, Chan-Ik, and Tae-Young Choe. "An optimal scheduling algorithm based on task duplication." In Proceedings. Eighth International Conference on Parallel and Distributed Systems. ICPADS 2001, pp. 9-14. IEEE, 2001.

[10] Topcuoglu, Haluk, Salim Hariri, and Min-You Wu. "Task scheduling algorithms for heterogeneous processors." In Proceedings. Eighth Heterogeneous Computing Workshop (HCW'99), pp. 3-14. IEEE, 1999.

[11] Koehler, Philip, Arun Anandasivam, and Dan MA. "Cloud services from a consumer perspective." AIS, 2010.

[12] Casas, Pedro, and Raimund Schatz. "Quality of experience in cloud services: Survey and measurements." Computer Networks 68 (2014): 149-165.

[13] Whitley, Darrell. "A genetic algorithm tutorial." Statistics and computing 4, no. 2 (1994): 65-85.

[14] Davis, Lawrence. "Handbook of genetic algorithms." (1991).

[15]Bäck, Thomas, David B. Fogel, and Zbigniew Michalewicz, eds. Evolutionary computation 1: Basic algorithms and operators. CRC press, 2018.

[16] Panda, Sanjaya K., and Prasanta K. Jana. "An efficient task consolidation algorithm for cloud computing systems." In International Conference on Distributed Computing and Internet Technology, pp. 61-74. Springer, Cham, 2016. 
Journal of trends in Computer Science and Smart technology (TCSST) (2019)

Vol.01/No.01

Pages: 46-56

https://irojournals.com/tcsst/

DOI: https://doi.org/10.36548/jtcsst.2019.1.005

[17] Al-Maamari, Ali, and Fatma A. Omara. "Task scheduling using PSO algorithm in cloud computing environments." International Journal of Grid and Distributed Computing 8, no. 5 (2015): 245-256. 